\title{
Elderly Fitz-Hugh-Curtis syndrome observed with superb microvascular imaging system
}

\author{
Tomotaka Yazaki $^{1} \cdot$ Shuichi Sato ${ }^{1,4} \cdot$ Hiroshi Tobita $^{1} \cdot$ Kazuki Isoda $^{1} \cdot$ Tatsuya Miyake $^{2} \cdot$ Yoshikazu Kinoshita $^{3}$
}

Received: 24 October 2017 / Accepted: 9 January 2018 / Published online: 20 February 2018

(c) The Author(s) 2018. This article is an open access publication

\begin{abstract}
Fitz-Hugh-Curtis syndrome (FHCS) is defined as inflammation on the surface of the liver following sexually transmitted chlamydia infection. We successfully observed the microvascular structure of the inflamed portion between the abdominal wall and surface of the liver in an elderly patient with FHCS using a superb microvascular imaging (SMI) system, a new technology developed for observing minute vascular flow. An 80-year-old Japanese female with right dorsal to lateral abdominal pain and fever came to our hospital. Anti-chlamydia antibodies were positive. SMI revealed signals suggesting small vessels passing from the liver surface to the hypoechoic space.
\end{abstract}

Keywords Liver $\cdot$ Fitz-Hugh-Curtis syndrome $\cdot$ Superb microvascular imaging $\cdot$ Elderly $\cdot$ Contrast-enhanced ultrasound

\section{Introduction}

Fitz-Hugh-Curtis syndrome (FHCS), defined as inflammation on the surface of the liver appearing as a result of sexually transmitted chlamydia infection, nearly exclusively occurs in women. Adhesions between the abdominal wall and surface of the liver following peri-inflammation of the liver sometimes cause right-side epigastric pain, with 'violin string sign' adhesions between the abdominal wall and liver surface a specific finding in a laparoscopic examination $[1,2]$. However, laparoscopy performed for diagnosis of FHCS is a slightly invasive procedure. Some recent reports have noted that imaging examination findings obtained with contrast-enhanced computed tomography (CECT) were useful for diagnosing FHCS [1, 2]. Here, we report successful observation of the microvascular structure in the inflamed

Shuichi Sato

bbsato@med.shimane-u.ac.jp

1 Department of Hepatology, Faculty of Medicine, Shimane University, Izumo, Japan

2 Department of Hepatology, Shimane Prefectural Hospital, Izumo, Japan

3 Department of Gastroenterology, Faculty of Medicine, Shimane University, Izumo, Japan

4 Department of Gastroenterology and Hepatology, Faculty of Medicine, Shimane University, 89-1 Enya-cho, Izumo, Shimane, Japan portion between the abdominal wall and surface of the liver in an elderly patient with FHCS using a superb microvascular imaging (SMI) system, a new technology developed for observing lower vascular flow that cannot be observed by conventional color Doppler imaging.

\section{Case report}

An 80-year-old Japanese woman with right dorsal to lateral abdominal pain and fever came to our hospital. Pyelonephritis was suspected based on the clinical course and pain location, and she was prescribed ceftriaxone sodium hydrate by the Department of Urology. Following that treatment, her symptoms did not improve, and she was transferred to our department. The patient had a history of hypertension, irritable cystic bladder, and transient ischemic attack. She had no habit of alcohol intake or smoking, and had not experienced sexual intercourse for several years.

Patient height was $158 \mathrm{~cm}$, body weight was $39.7 \mathrm{~kg}$, and body temperature was $37.9{ }^{\circ} \mathrm{C}$. Blood pressure and heart rate were also abnormal at $164 / 96 \mathrm{mmHg}$ and $81 / \mathrm{min}$, respectively. There was no remarkable physical finding except for tenderness in the right dorsal and lateral abdominal areas. The liver, spleen, and superficial lymph nodes were not palpable.

Laboratory findings on admission showed mild elevation of biliary enzymes and renal dysfunction. 
Anti-chlamydia IgA and IgG antibodies were positive, while serum anti-gonococcus antibody and interferongamma release assay findings were negative (Table 1). CECT showed enhancement of the liver surface and a slight fluid space surrounding the liver (Fig. 1). An ultrasonography examination with an Aplio 500 ${ }^{\mathrm{TM}}$ (Toshiba, Co., Ltd, Tokyo; PVT-375BT 6C1 convex probe) using $\mathrm{B}$-mode showed a thin hypoechoic but not echo-free space between the abdominal wall and surface of the liver (Fig. 2a). SMI results revealed signals suggesting small vessels passing from the liver surface to the hypoechoic space on the liver surface (Fig. 2b). Furthermore, contrast-enhanced ultrasonography (CEUS) with Sonazoid ${ }^{\circledR}$ also showed a stream of microbubbles from the liver parenchyma to the hypoechoic space (Fig. 2c).

Positive results for anti-chlamydia IgA and IgG antibodies, as well as ultrasonography and CT findings, suggested FHCS in an acute phase. Administration of azithromycin and cephalosporin temporarily improved serum CRP and symptoms. However, serum CRP rebounded and symptom relapse occurred several days after the end of the antibiotics treatment, which were improved by continuous administration of minocycline. Bacterial findings from a gynecological vaginal examination did not detect chlamydia or gonococcus. On hospital day 150, an ultrasonography examination showed an absence of the hypoechoic space on the liver surface (Fig. 3). At that time, the concentration of anti-chlamydia antibodies was slightly higher as compared to prior to beginning treatment for FHCS (anti-chlamydia IgG cut-off index (COI) 2.765, anti-chlamydia IgA COI 8.746). Fifteen months after FHCS onset, the patient had no abdominal symptoms and serum CRP was $0.02 \mathrm{mg} / \mathrm{dL}$ (Fig. 4).

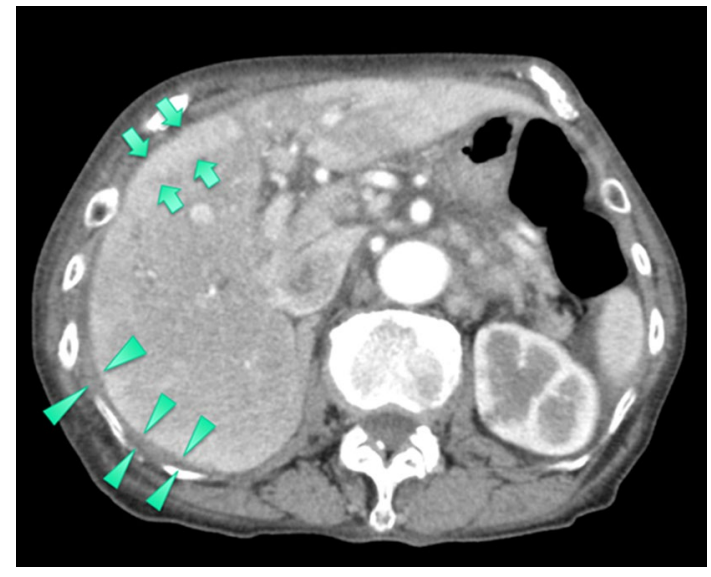

Fig. 1 CECT image showing enhancement of the liver surface (arrows) and a small fluid space surrounding the liver (arrowheads)

\section{Discussion}

FHCS was initially reported by Curtis et al. in open surgery observation as adhesions between the abdominal wall and surface of the liver, and then 4 years later Fitz-Hugh reported peri-hepatitis and salpingitis in association with gonococcal infection [1-4]. Later in 1982, Wolner-Hassen reported a case in which chlamydia infection caused FHCS [3]. Presently, the most common cause of FHCS is thought to be infection by chlamydia, the primary bacterium in cases of sexually transmitted infection [4]. More than $90 \%$ of cases of uterine cervicitis associated with chlamydia infection are asymptomatic, though when left untreated can lead to endometritis and pelviperitonitis, with the final result perihepatitis [5]. The most susceptible age for FHCS is approximately 20 years, though cases of elderly patients with FHCS caused by reactivation of chlamydia have been reported [6].
Table 1 Laboratory findings on admission

\begin{tabular}{llll}
\hline WBC & $12,100(1 / \mu \mathrm{L})$ & BUN & $11.3(\mathrm{mg} / \mathrm{dL})$ \\
RBC & $3.73 \times 10^{6}(1 / \mu \mathrm{L})$ & Crea. & $0.38(\mathrm{mg} / \mathrm{dL})$ \\
$\mathrm{Hb}$ & $11.3(\mathrm{~g} / \mathrm{dL})$ & CRP & $16.5(\mathrm{mg} / \mathrm{dL})$ \\
Platelets & $326 \times 10^{3}(1 / \mu \mathrm{L})$ & PCT & $0.23(\mathrm{ng} / \mathrm{mL})$ \\
$\%$ prothrombin & $65.7(\%)$ & CEA & $1.9(\mathrm{ng} / \mathrm{mL})$ \\
APTT & $33.7(\mathrm{~s})$ & CA19-9 & $8.0(\mathrm{U} / \mathrm{mL})$ \\
T.P. & $6.9(\mathrm{~g} / \mathrm{dL})$ & & \\
Albumin & $2.9(\mathrm{~g} / \mathrm{dL})$ & Anti-chlamydia antibody $\operatorname{IgG}(+)$ & $2.547 \mathrm{COI}$ \\
T. bilirubin & $0.7(\mathrm{mg} / \mathrm{dL})$ & Anti-chlamydia antibody IgA $(+)$ & $6.103 \mathrm{COI}$ \\
AST & $28(\mathrm{U} / \mathrm{L})$ & Anti-gonococcus antibody $(-)$ & \\
ALT & $19(\mathrm{U} / \mathrm{L})$ & Interferon-gamma release assay $(-)$ & \\
LDH & $155(\mathrm{U} / \mathrm{L})$ & Chlamydia antigen $(-)$ & \\
ALP & $469(\mathrm{U} / \mathrm{L})$ & & \\
GGT & $15(\mathrm{U} / \mathrm{L})$ & & \\
\hline
\end{tabular}



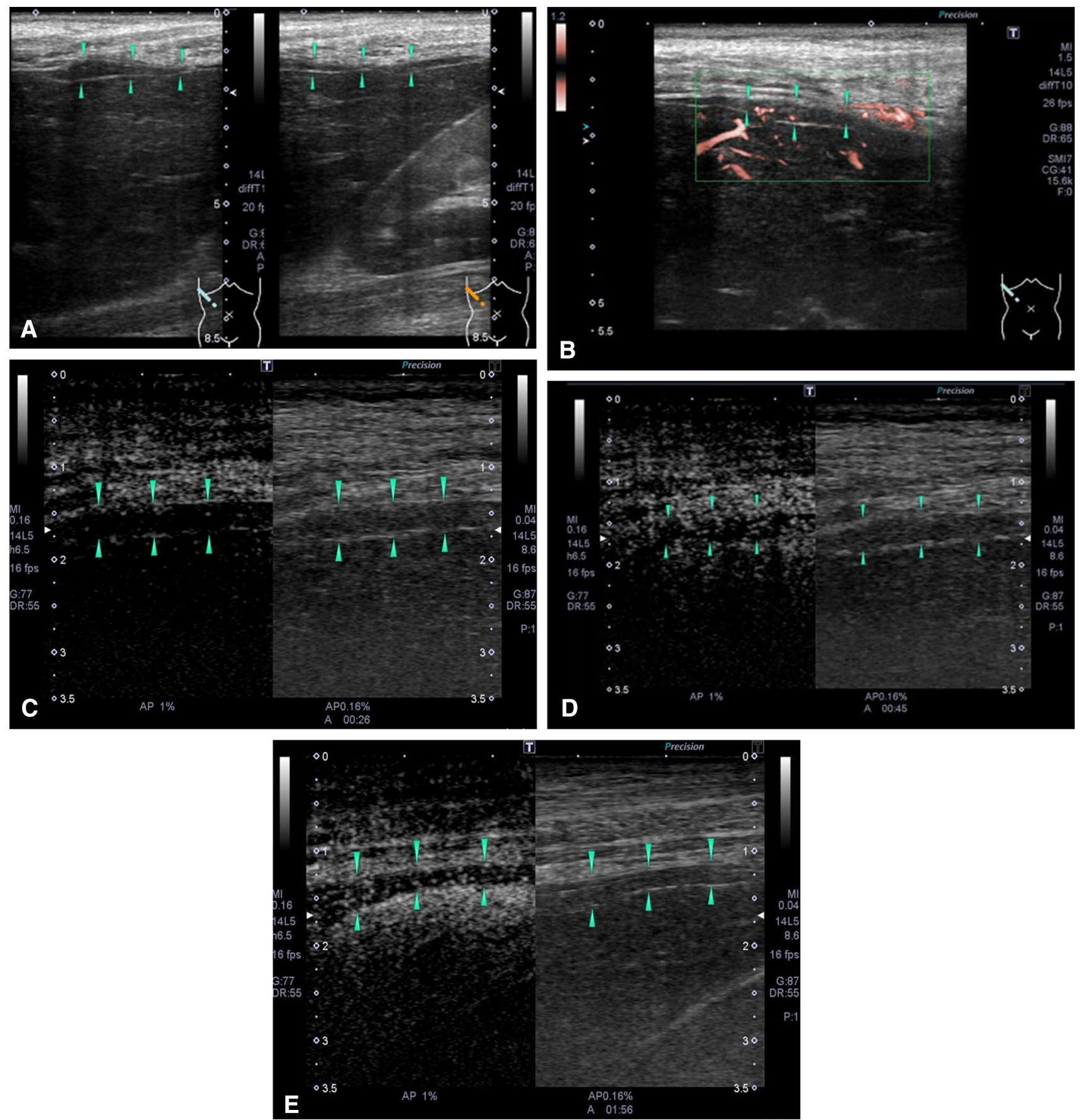

Fig. 2 Ultrasonography findings. a Right intercostal view with B-mode showing a thin hypoechoic space between the abdominal wall and liver surface (arrowheads). b SMI right intercostal view showing small blood flow signals passing from the liver surface to the hypoechoic space on the liver surface (arrowheads). CEUS with Sonazoid $^{\circledR}$ right intercostal view showing a stream of microbubbles from the liver parenchyma to the hypoechoic space in the vascular phase (arrowheads). c CEUS image obtained $26 \mathrm{~s}$ after administration

A diagnosis of FHCS is made following detection of perihepatitis or fibrous adhesion between the abdominal wall and liver surface, so-called 'violin string sign', in laparoscopy of Sonazoid ${ }^{\circledR}$. Microbubbles are slightly evident in the hypoechoic space and on the liver surface. d CEUS image obtained $45 \mathrm{~s}$ after administration of Sonazoid ${ }^{\circledR}$. Microbubbles are clearly evident in the hypoechoic space and on the liver surface. e CEUS image obtained $116 \mathrm{~s}$ after administration of Sonazoid ${ }^{\circledR}$. Microbubbles are increased on the surface of the liver, while there is no change in the hypoechoic space

or surgery findings. An invasive examination to diagnose FHCS tends to be avoided, because most affected patients are young females. Recently, CECT has been found to be 
Fig. 3 Ultrasonography examination findings. Right intercosSMI obtained on hospital day 150 . No hypoechoic space is evident on the liver surface tal view with $\mathbf{a} \mathrm{B}$-mode and $\mathbf{b}$
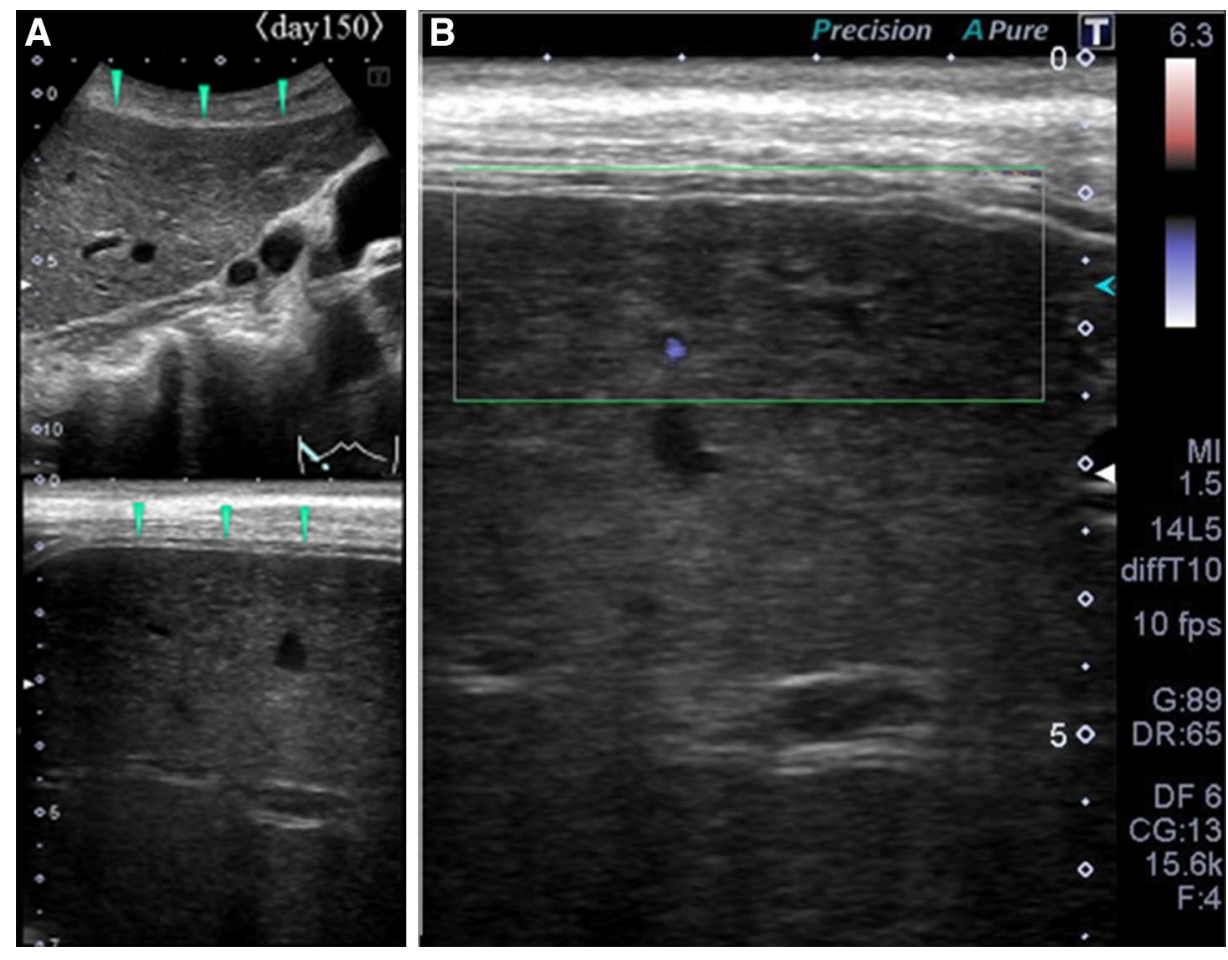

Fig. 4 Relationships among C-reactive protein (CRP), clinical course of symptoms, and period of antibiotics administration. $C M Z$ cefmetazole, $A Z M$ azithromycin, $M I N O$ minocycline

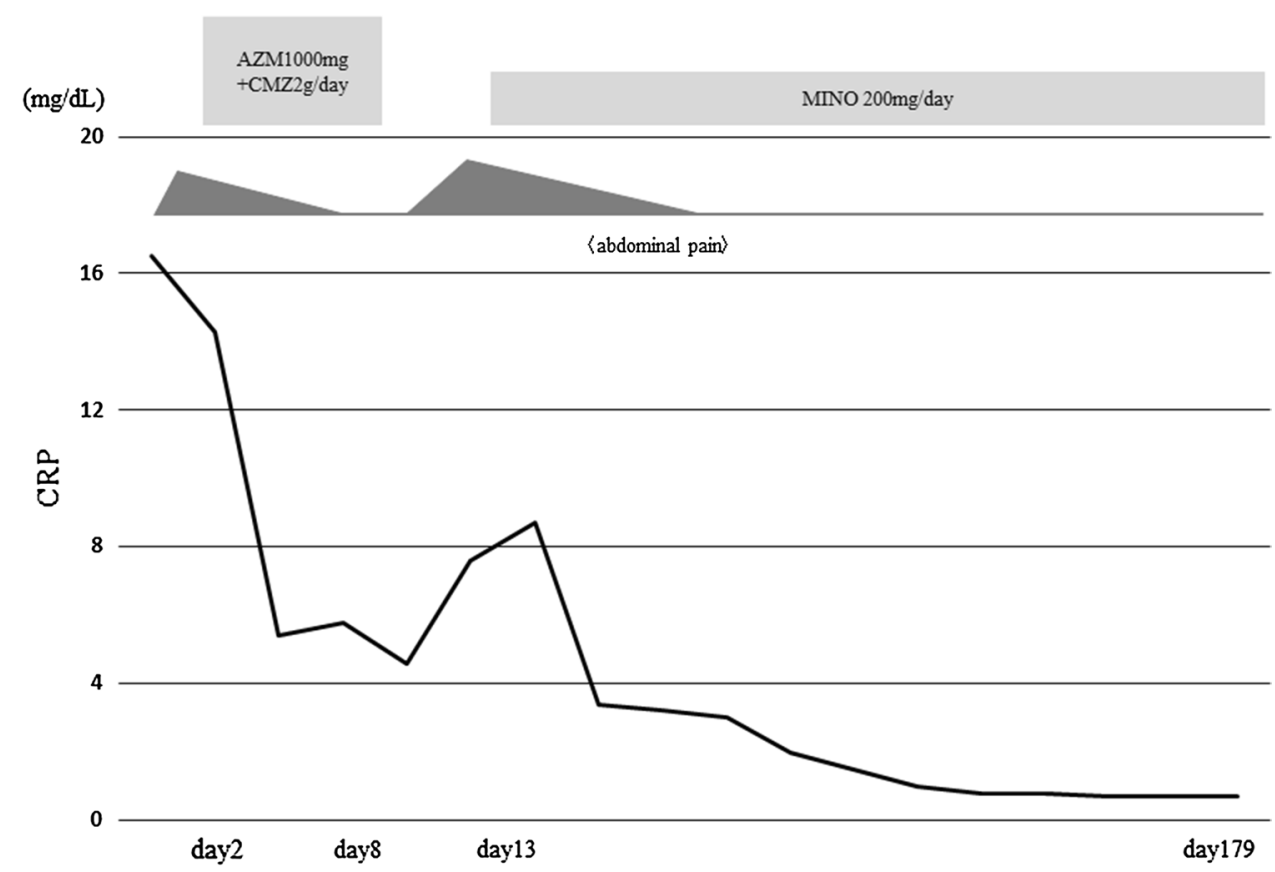

useful as a noninvasive and supplemental diagnostic tool [1, 7]. However, enhancement of the surface of the liver is only detected during the early arterial phase of CECT in patients with FHCS caused by chlamydia infection.

The final diagnosis of FHCS in the present case was difficult, because we were unable to directly observe the liver surface by laparoscopy, there was no histological confirmation, and the patient had not experienced sexual intercourse for several years. Nevertheless, right-side abdominal symptoms, enhancement of the liver surface in the early arterial phase of CECT, a gradual elevation of the chlamydia marker in serum, and response to antibiotic medication specific to chlamydia were helpful for 
diagnosing FHCS. In addition to those, SMI and CEUS findings ultimately contributed to confirm the FHCS diagnosis.

Fitz-Hugh-Curtis syndrome (FHCS) is generally known to commonly occur in women of childbearing age who do not use oral contraceptives and have sexual partners who are older than 25 years of age [8]. However, FHCS without presentation of sexually transmitted diseases has been seen in recent years. Furthermore, cases with C. trachomatis survival and reactivation under stressful conditions from production of IFN- $\gamma$ persistence have also been reported [9]. In our patient, the concentrations of these antibodies were slightly increased following treatment. A continuous increase in antibody titer may suggest a change after infection or reactivation of chlamydia, as well as change in IgGtype antibody concentration in serum following another viral infection.

The SMI system incorporated in Toshiba's Alpio ${ }^{\mathrm{TM}} 500$ ultrasound device is an innovative and unique ultrasound Doppler tool that employs a unique algorithm for visualization of minute vessels to show velocity without use of contrast media. Some recent reports have also noted that SMI is useful for assessing minute vascular flow in patients with carpal tunnel syndrome [10] and breast lesions [11]. SMI is also useful for visualizing the small vascular architecture of the liver surface associated with progression of fibrosis in patients with chronic hepatitis C [12]. Moreover, it has been demonstrated that SMI can clearly reveal not only spoke-wheel feeding arteries but also drainage hepatic veins in patients with hepatic focal nodular hyperplasia [13]. Conventional color Doppler imaging is not able to detect Doppler signals in a hypoechoic space existing between the abdominal wall and surface of the liver, and is only useful for suggesting the presence of infectious ascites or a hematoma on the liver surface. On the other hand, SMI findings can be used to rule out ascites and hematoma, as it is able to detect Doppler signals suggesting small vessels passing from the liver surface to a hypoechoic space on that surface. CEUS with Sonazoid can be used to confirm findings obtained with SMI.

In the present case, positive findings for anti-chlamydia IgA and IgG antibodies, and remarkable clinical response following administration of minocycline, ruled out the possibility of malignant abdominal mesothelioma, peritonitis carcinomatosa, tuberculous peritonitis, liver abscess rupture, and advanced cholecystitis, for which ultrasonography findings resemble those seen in FHCS [14].

In conclusion, we experienced a rare case of FHCS in an elderly patient, for which SMI was useful for observing the microvascular structure related to inflammation between the abdominal wall and surface of the liver.

\section{Compliance with ethical standards}

Conflict of interest None declared.

Ethical statements Additional informed consent was obtained from the patient for whom identifying information is included in this article.

Open Access This article is distributed under the terms of the Creative Commons Attribution 4.0 International License (http://creativeco mmons.org/licenses/by/4.0/), which permits unrestricted use, distribution, and reproduction in any medium, provided you give appropriate credit to the original author(s) and the source, provide a link to the Creative Commons license, and indicate if changes were made.

\section{References}

1. Curtis AH. A cause of adhesion in the right upper quadrant. J Am Med Assoc. 1930;94:1221-2.

2. Fitz-Hugh T. Acute gonococcic peritonitis of the right upper quadrant in women. J Am Med Assoc. 1934;102:2094-6.

3. Wølner-Hanssen P, Svensson L, Weström L, et al. Isolation of Chlamydia trachomatis from the liver capsule in Fitz-Hugh-Curtis syndrome. N Engl J Med. 1982;113:306.

4. Cates W Jr, Wasserheit JN. Genital chlamydial infections: epidemiology and reproductive sequelae. Am J Obstet Gynecol. 1991;164:1771-81.

5. Peter NG, Clark LR, Jaeger JR. Fitz-Hugh-Curtis syndrome: a diagnosis to consider in women with right upper quadrant pain. Cleve Clin J Med. 2004;71:233-9.

6. Oh SN, Rha SE, Byun JY, et al. Chilaiditi syndrome caused by Fitz-Hugh-Curtis syndrome: multidetector CT findings. Abdom Imaging. 2006;31:45-7.

7. Cho HJ, Kim HK, Suh JH, et al. Fitz-Hugh-Curtis syndrome: CT findings of three cases. Emerg Radiol. 2008;15:43-6.

8. Yi H, Shim S, Kim G, et al. Case of Fitz-Hugh-Curtis syndrome in male without presentation of sexually transmitted disease. World J Clin Cases. 2015;3:965-9.

9. Muramatsu MK, Brothwell JA, Stein BD, et al. Beyond tryptophan synthase: identification of genes that contribute to Chlamydia trachomatis survival during gamma interferon-induced persistence and reactivation. Infect Immun. 2016;84:2791-801.

10. Chen J, Chen L, Wu L, et al. Value of superb microvascular imaging ultrasonography in the diagnosis of carpal tunnel syndrome: compared with color Doppler and power Doppler. Medicine (Baltimore). 2017;96:e6862.

11. Ma Y, Li G, Li J, et al. The diagnostic value of superb microvascular imaging (SMI) in detecting blood flow signals of breast lesions. Medicine (Baltimore). 2015;94:e1502.

12. Kuroda H, Abe T, Kakisaka K, et al. Visualizing the hepatic vascular architecture using superb microvascular imaging in patients with hepatitis $\mathrm{C}$ virus: a novel technique. World J Gastroenterol. 2016;22:6057-64.

13. Naganuma $H$, Ishida $H$, Ogawa $M$, et al. Visualization of draining vein in focal nodular hyperplasia by superb microvascular imaging: report of two cases. J Med Ultrason. 2017;44:323-8.

14. Kim S, Kim TU, Lee JW, et al. The perihepatic space: comprehensive anatomy and CT features of pathologic conditions. Radiographics. 2007;27:129-43. 